\title{
ARTICLE \\ Repetitive transcranial magnetic stimulation targeting the insular cortex for reduction of heavy drinking in treatment-seeking alcohol-dependent subjects: a randomized controlled trial
}

\author{
Irene Perini ${ }^{1}$, Robin Kämpe ${ }^{1}$, Theodor Arlestig ${ }^{1}$, Hanna Karlsson ${ }^{1,2}$, Andreas Löfberg ${ }^{1,2}$, Michal Pietrzak ${ }^{1}$, Abraham Zangen ${ }^{3}$ and \\ Markus Heilig ${ }^{1,2}$
}

Insula responses to drug cues are correlated with cravings, and lesions in this area reduce nicotine seeking. Here, we investigated the potential efficacy of repetitive transcranial magnetic stimulation (rTMS) targeting the insula in alcohol addiction. Treatmentseeking alcohol-dependent patients (Diagnostic and Statistical Manual of Mental Disorder, Fourth Edition; $N=56$ ) participated in this double-blind, sham-controlled, randomized trial. Participants received $10 \mathrm{~Hz}$ rTMS or sham using an $\mathrm{H} 8$ coil, 5 days a week for 3 weeks. Stimulation targeted insular cortex and overlaying regions bilaterally, while excluding anterior prefrontal areas. Craving and self-reported as well as biomarker-based drinking measures were collected at baseline, during treatment, and through 12 weeks. Resting-state magnetic resonance imaging (rsMRI) data were collected before and after treatment. Task-based MRI was used to probe brain correlates of reward processing, affective responses, and alcohol following completion of treatment. A marked overall decrease in craving and drinking measures was observed during treatment, but did not differ between rTMS or sham stimulation. Both groups equally increased their alcohol use following completion of treatment and through the 12-week follow-up. Analysis using seeds in the insula identified differences in resting-state connectivity between active and sham groups at completion of treatment, potentially indicating an ability of treatment to modify insula function. However, while each task robustly replicated brain responses established in the literature, no effects of rTMS were found. Collectively, this study does not support efficacy of rTMS targeting the insula in alcohol addiction.

Neuropsychopharmacology (2020) 45:842-850; https://doi.org/10.1038/s41386-019-0565-7

\section{INTRODUCTION}

Alcohol dependence (equivalent to moderate-severe alcohol use disorder according to Diagnostic and Statistical Manual of Mental Disorder, Fifth Edition [1]; hereafter equated with alcohol addiction, or simply alcoholism) has a global prevalence of $2.6 \%$ (WHO 2018). Although alcoholism treatments with documented beneficial effects exist, their effect sizes are limited $(\sim d=0.3)$ $[2,3]$, and relapse rates remain high ( 65\% [4]).

Behavioral treatments remain the mainstay of alcoholism treatment, while medical treatments are limited to pharmacotherapies that meet marginal patient and practitioner uptake, and are prescribed to a small minority of patients [5]. Device-based therapies may offer this treatment toolkit to be expanded. In particular, non-invasive neuromodulation using repetitive transcranial magnetic stimulation (rTMS) has recently demonstrated efficacy in some neuropsychiatric disorders [6-8]. Paralleling those findings, rTMS has also been suggested to hold therapeutic potential in addictive disorders [9, 10]. Data have provided initial support to the efficacy of this modality in nicotine addiction [11-14]. In other substance use disorders, including alcohol addiction, available data are more limited and inconclusive.

Prior studies to evaluate rTMS in alcohol addiction have focused on the dorsolateral prefrontal cortex (dIPFC). A significant reduction in alcohol craving over the course of 1 month was initially reported following rTMS to the right dIPFC (10 sessions, $10 \mathrm{~Hz}$ ) compared to sham [15]. However, another sham-controlled study that used 10 sessions of $20 \mathrm{~Hz}$ rTMS targeting the left dIPFC in alcohol-dependent women failed to find any effect on alcohol craving or mood [16]. More recently, two double-blind sham-controlled pilot studies targeting dIPFC bilaterally [17] and medial PFC bilaterally [18] reported reductions in craving and drinking in patients with alcohol dependence, with effects lasting 1-6 months. These data must, however, be considered as highly preliminary, given the small sample sizes (5 and 9, respectively).

In summary, while available evidence suggests that multisession, high-frequency rTMS merits evaluation for efficacy in alcohol addiction [19], findings to date are inconclusive at best $[13,20]$. Randomized, sham-controlled studies that use larger

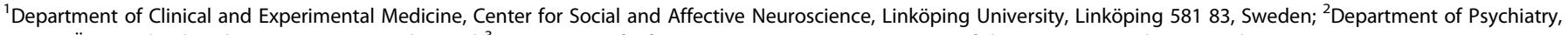
Region Östergötland, Linköping 581 85, Sweden and ${ }^{3}$ Department of Life Sciences, Ben-Gurion University of the Negev, Beer Sheva, Israel

Correspondence: Irene Perini (irene.perini@liu.se)

These authors contributed equally: Irene Perini, Robin Kämpe

Received: 8 July 2019 Revised: 8 October 2019 Accepted: 1 November 2019

Published online: 11 November 2019 
sample sizes and obtain objective measures of alcohol use are needed.

We therefore set out to carry out a double-blind, shamcontrolled randomized rTMS study in alcoholism. We chose to target the insular cortex, a region whose activity is strongly associated with craving [21], and which has been successfully targeted in nicotine addiction [12]. Several studies have reported the involvement of the insula in cravings for food, cocaine, and cigarettes [22-24], as well as in decision-making processes [25]. The possibility that the link between insula and craving is causal rather than merely correlational is suggested by the fact that damage to the insula results in higher probability of quitting smoking, compared to brain damage in other regions [26]. Accordingly, a recent "deep" rTMS study using the "H-coil" design targeting the insula (but also prefrontal regions) bilaterally showed promising effects in nicotine addiction [12].

Here, we evaluated whether rTMS targeting the insula would be beneficial in alcohol addiction. Treatment-seeking alcohol-dependent participants were randomized to active $10 \mathrm{~Hz}$ rTMS or sham stimulation using a custom-made $\mathrm{H} 8$ coil that targeted the insula and the overlaying regions, while excluding anterior prefrontal areas and ensuring a more selective stimulation relative to the $\mathrm{H} 4$ coil used in the above-mentioned study [12]. Treatment was 5 days a week for 3 weeks, and outcome measures were collected for 12 weeks post treatment. Outcomes included craving and drinking self-reports, but also objective biomarkers of consumption. To address mechanistic questions, we also obtained posttreatment cerebrospinal fluid (CSF) samples, and both pre- and post-treatment magnetic resonance imaging (MRI) scans. Structural and resting-state scans were collected before and after treatment. Post-treatment scan also included tasks to investigate the impact of rTMS on reward processing, assessed with the classic monetary incentive delayed (MID) paradigm [27], and processing of negative affect and responses to alcohol-related pictures, using picture-matching paradigms modified from Hariri et al. [28].

\section{MATERIALS AND METHODS}

Participants

Fifty-six treatment-seeking alcohol-dependent patients (24 recruited from the addiction clinics, 32 recruited by newspaper advertisement and flyers) participated in this double-blind, randomized, sham-controlled, clinical trial. Eligible participants were formally included after providing a written informed consent and were then randomly assigned to either sham or $10 \mathrm{~Hz}$ rTMS with a 1:1 allocation ratio. Twenty nine were allocated to active rTMS stimulation and 27 were assigned the sham (Fig. 1).

Detailed eligibility criteria are provided at ClinicalTrials.gov (NCT02643264). Inclusion criteria included current Diagnostic and Statistical Manual of Mental Disorders, Fourth Edition [29] diagnosis of alcohol dependence, alcohol use during the last month, age between 25-64 years, and right-handedness. Female participants were included if postmenopausal, or following a negative urine pregnancy test and when using an effective birth control method. If clinically indicated, prospective participants were treated for acute alcohol withdrawal according to standard clinical guidelines at the addiction clinic before screening.

Exclusion criteria included more than mild cognitive impairment, assessed using the Mini Mental State Examination (MMSE < 24; [30]). Schizophrenia, bipolar, or other psychotic disorder, any clinically significant neurological disorder or lesion, hearing impairment, or pregnancy were also exclusion criteria. If there were reports of seizure-like events that were not confirmed by the medical records, participants were clinically evaluated by a clinical neurophysiology consult using electroencephalogram (EEG). Other exclusion criteria were use of illicit drugs or medications known to increase the risk for seizures, as defined by international

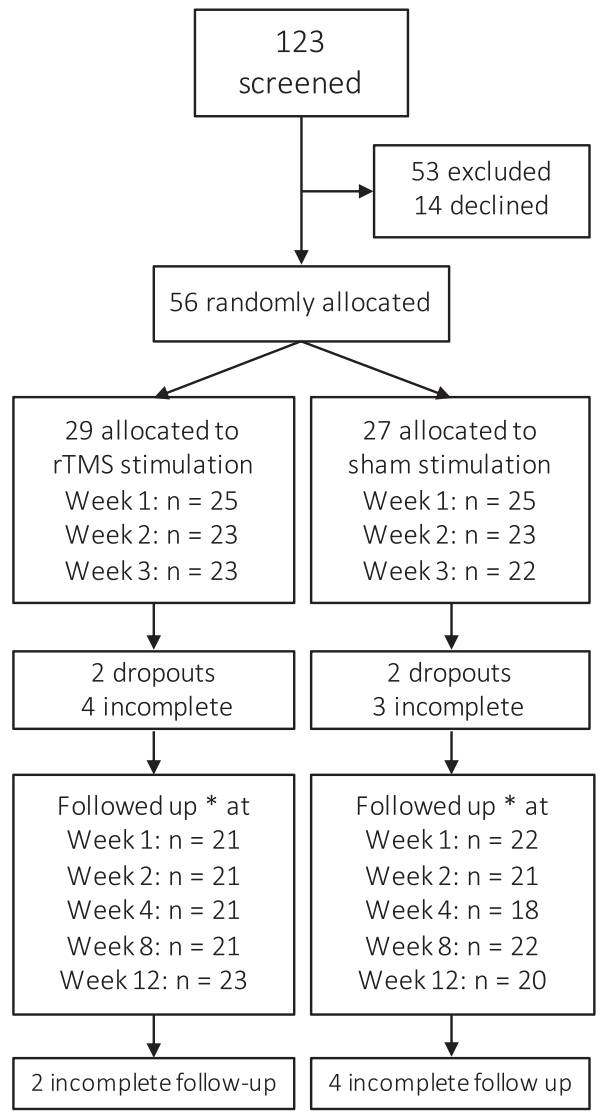

Fig. 1 Consort diagram of study participants. *Reporting TLFB on the last follow-up visit

consensus guidelines for TMS delivery [31], as well as inability to participate in an MRI scan, assessed using an MRI checklist.

Participants were recruited at the addiction and psychiatry clinic of Linköping University Hospital, Sweden, using flyers handed out at treatment locations and by advertisement in the local newspaper. Recruitment was ongoing between September 2015 and October 2018. The study was approved by the Regional Ethical Board of Linköping (ref. no. 2015/130-31), and all participants provided written informed consent.

Baseline assessments: Psychiatric interview and personality traits. Alcohol dependence severity was assessed using the Alcohol Use Disorder Identification Test (AUDIT) [32], the Alcohol Dependence Scale (ADS) [33], and the Addiction Severity Index (ASI) [34, 35], which measures the impact of addiction on medical and psychosocial functioning. Psychiatric evaluation, including a formal alcohol diagnosis, was performed using the Structured Clinical Interview for DSM diagnosis (SCID [36]). Severity of depression, anxiety, and compulsion symptoms was obtained by self-report using the Comprehensive Psychopathological Rating Scale, SelfRate (CPRS-SA [37]) and the Clinical Global Impression (CGI [38]). Baseline personality traits were obtained using the Abbreviated Personality Assessment (NEO-FFI, [39]).

\section{Study timeline}

The study consisted of three phases: screening, treatment, and follow-up (Table 1). During the screening phase, prospective participants were evaluated for eligibility for up to 14 days by a psychiatric research nurse. After inclusion and before treatment, participants' structural MRI and resting-state scans were collected. 
Table 1. Study timeline and assessment

\begin{tabular}{|c|c|c|c|c|c|c|c|c|c|}
\hline & Screening & \multicolumn{3}{|c|}{ Treatment } & \multicolumn{5}{|c|}{ Follow-up } \\
\hline \multicolumn{10}{|l|}{ Method } \\
\hline rTMS & & $\cdot$ & • & • & & & & & \\
\hline MRI & & - & & - & & & & & \\
\hline $\mathrm{LP}$ & & & & - & & & & & \\
\hline ADS & $\cdot$ & & & & & & & & \\
\hline PEth & $\cdot$ & - & • & - & - & • & • & • & • \\
\hline TLFB & $\cdot$ & & & $\cdot$ & $\cdot$ & $\cdot$ & $\cdot$ & $\cdot$ & $\cdot$ \\
\hline \multicolumn{10}{|l|}{ Craving } \\
\hline$A \cup Q$ & & $\cdot$ & - & $\cdot$ & & & & & \\
\hline SCID & $\cdot$ & & & & & & & & \\
\hline CRPS-SA & $\cdot$ & $\cdot$ & $\cdot$ & $\cdot$ & • & $\cdot$ & $\cdot$ & $\cdot$ & • \\
\hline CGI & & $\cdot$ & $\cdot$ & $\cdot$ & $\cdot$ & $\cdot$ & • & $\cdot$ & $\cdot$ \\
\hline NEO-FFI & $\cdot$ & & & & & & & & \\
\hline ASI & $\cdot$ & & & & & & • & • & $\cdot$ \\
\hline SETS & & & & & - & & & & \\
\hline COT/CRE & • & & & & $\cdot$ & • & • & - & - \\
\hline
\end{tabular}

rTMS treatment parameters were based on an earlier deep rTMS study in smokers [12]; however, while that study targeted the anterior insula and PFC using the H4 dTMS coil, the present study targeted the insula excluding anterior prefrontal regions using the $\mathrm{H} 8$ coil. During the treatment phase, which lasted for 3 weeks, participants received one stimulation session daily for 5 days a week, for a total of 15 treatment sessions. After the last treatment session, participants underwent an additional MRI session, where structural, resting-state, and task-based scans were collected. Finally, participants underwent an optional lumbar puncture procedure where CSF was collected (see Supplementary Material). Participants returned for follow-up visits 1, 2, 4, 8, and 12 weeks after last treatment to evaluate effects on alcohol use. To assess effects of rTMS on nicotine consumption, we extracted cotinine/creatine ratio ("COT/CRE") in urine at baseline and followup sessions.

rTMS stimulation

Deep rTMS was delivered via a Magstim Rapid magnetic stimulator (Magstim, Whitland, Dyfed, UK) bilaterally over the insula, using an "H8 coil" (Brainsway, Jerusalem, Israel), which is designed to allow for stimulation of deeper brain regions than regular "figure-of-eight" coils [40,41]. The optimal spot on the scalp for stimulation of the right motor cortex was localized and resting motor threshold (MT) was defined using the right component of the $\mathrm{H} 8$ coil before the first session and repeated every week. The MT is defined as the minimum intensity that with a $\geq 50 \%$ probability elicits a muscle response of the left abductor pollicis brevis; this measure is used to determine the treatment parameters to balance efficacy and safety [31]. The coil positioning was tailored to participants' head size, longitudinally at $40 \%$ of nasion-inion distance. The lateral-medial positioning was tailored to ear to ear distance over the head, by subtracting ear canal distance from $52 \mathrm{~cm}$ to a measuring tape strap located inside the coil. Each stimulation train consisted of 30 pulses, lasted $3 \mathrm{~s}$, and was delivered at an intensity of $120 \%$ of the MT as measured with the right component of the coil, with a frequency of $10 \mathrm{~Hz}$. A total of 50 trains (1500 pulses) were delivered, with a 20 -s inter-train interval, as described previously [12]. There was about $10 \%$ difference in stimulator output during unilateral thresholding and bilateral active stimulation, with latter being lower. The resulting electric field intensity at various relevant measures of depth was measured in a model of the human head $\left(15 \times 13 \times 18 \mathrm{~cm}^{3}\right)$, filled with physiologic saline solution. Average MT corresponded to $60 \%$ of maximum stimulator output, and resulted in an electric field of $138 \mathrm{~V} / \mathrm{m}$ on the cortical surface. At $5 \mathrm{~mm}$ depth from the lateral edge of the insula (point A: MNI coordinates $x=39, y=6, z=14$ ), the stimulation induced an electric field of $79 \mathrm{~V} / \mathrm{m}$. At a point in the insular most lateral edge (MNI coordinates $x=44, y=6, z=$ $14)$, the induced electric field was $89 \mathrm{~V} / \mathrm{m}$. The $\mathrm{H} 8$ coil was placed over the theoretical frontal cortex of the head model and the field in each pixel was measured using a "pick-up" dipole probe, attached to an oscilloscope. To inspect potential effects of stimulation strength and drinking outcome, individual percentage stimulation strength values, based on maximum stimulator output, were extracted per participant receiving active stimulation and regressed on delta phosphatidylethanol (PEth [42]) and delta timeline follow back (TLFB [43]) scores. Delta scores reflected the difference between scores at the end of treatment compared to screening.

Sham treatment was performed using the H8-coil sham settings (encased in the same helmet with the active coil), which was designed to mimic the auditory artifact and the scalp sensations evoked by the active coil, without stimulating the brain itself. Randomization of the respective operation mode of the stimulation $(10 \mathrm{~Hz}$ or sham) was determined by a pre-programmed magnetic treatment card individually assigned to each subject through the double-blind randomization process. Participants, operators, and the medical staff who collected assessments were all blinded to stimulation type. Blood pressure and pulse were 
monitored every 5 min during the treatment. When participants exhibited strong facial twitches, they were provided with dental cotton rolls to reduce discomfort.

Every rTMS session was preceded by a presentation of an alcohol cue, since cue presentation was suggested to increase the efficacy of rTMS in the nicotine trial on which our stimulation parameters were patterned [12]. Participants were asked to first pour a glass of water from a container, smell/handle the content of the glass for $3 \mathrm{~min}$, and refrain from consuming it. They were then asked to repeat the same sequence with their pre-selected preferred alcohol beverage. The self-report craving measure Acute Urge Questionnaire (AUQ [44]) was obtained after water and alcohol cue exposure during each treatment day. To assess whether participants believed they received active treatment or sham, participants completed the Stanford Expectations of Treatment Scale (SETS) [45].

Psychometric and physiological measures

Table 1 shows the study timeline with assessment timepoints.

Alcohol consumption. Serum PEth [42] was assessed by the SWEDAC accredited clinical chemistry laboratory at Linköping University Hospital, and was used as a highly specific, sensitive, and quantitative biomarker of alcohol use. PEth is a degradation product in the blood that only forms in the presence of alcohol. Using the accredited analysis, concentrations $<0.05 \mu \mathrm{mol} / \mathrm{L}$ indicate abstinence; those between 0.05 and 0.30 indicate moderate use, while levels exceeding $0.3 \mu \mathrm{mol} / \mathrm{L}$ are considered to reflect heavy alcohol use. In addition, measures of use were obtained using the TLFB self-report, a retrospective estimation of drinking during the past 91 days at screening, during the past 21 at end of treatment, and during the period preceding each follow-up visits [43].

Alcohol craving. Craving for alcohol was obtained using the AUQ, which addresses acute craving for alcohol at the moment of the assessment and was administered each treatment day before rTMS or sham stimulation. In addition, the Penn Alcohol Craving Scale (PACS [46]), which reflects estimates of alcohol craving during the previous week, was administered once a week during treatment and during each follow-up sessions.

Statistical analysis. For dependent measures, individual scores for each subject were entered in repeated-measures analysis of variance (ANOVAs), with "time" as within-subject factor and "group" as between-subject factor (two levels: rTMS and sham) when appropriate. For pairwise comparisons, independent-sample $T$ tests or Mann-Whitney were performed depending on assumption of normality of the data. The number of levels for the ANOVA's within-subject factor depended on the number of assessments per measure (Table 1). For craving and drinking measures that included treatment and follow-up assessments, analyses were performed separately during treatment and followup sessions and in both cases included screening scores, when available. With "treatment" we refer to the 15 days of stimulation period, disregarding whether the stimulation was rTMS of sham. When sphericity was violated according to Maunchly's test for sphericity, Greenhouse-Geisser's correction was implemented. Analysis of follow-up measures controlled for participants on disulfiram (Antabus). All behavioral measures were analyzed using the IBM Statistical Package for the Social Sciences (SPSS) version 25 and graphs were made in GraphPad Prism8.

MRI procedure

MRI data preprocessing and analysis. MRI data acquisition, preprocessing, and analysis details are presented in the Supplementary Material. Preprocessing and analysis were performed with the Analysis of Functional Neuro Images (AFNI) software v.18.3.16 [47]. To achieve multiple comparison corrected results not suffering from inflated false positives [48], activation maps were thresholded at a per-voxel $P$ value of 0.002 together with a cluster $a$ of 0.05 , in accordance with current stringent AFNI approach [49]. For resting-state data, full-brain connectivity analysis was performed on six insula seed locations identified by maximizing the bilateral connectivity of each seed location (see Supplementary Material, Table S2). To address whether individual anatomical differences in insular depth affected drinking and resting-state $\mathrm{fMRI}$ outcome, we calculated the insular-to-scalp distance and regressed on delta PEth, delta TLFB, and resting-state $\beta$ correlation coefficients. Insular depth was calculated in each participants receiving active stimulation using the Image Processing and Analysis in Java (ImageJ) software, version 1.5 [50]. Using T1 images in axial view, we averaged the distance between the insular central gyrus and the scalp on right and left sides.

Power and interim analyses

To estimate target participant number, a power analysis was performed, assuming a repeated-measures model on the primary drinking outcome PEth. The model included four within-subjects measures (screening, treatment w1, treatment w2, treatment w3) and a between-subject factor (rTMS, sham). Based on these assumptions, the study was designed to detect a medium or greater effect size, that is, Cohen's $f \geq 0.25$, with a power $\geq 0.80$ at $a=0.05$, at a sample size of 41 subjects per group. When approximately half of the total required sample size was collected, an interim analysis was performed on PEth scores. Effect size for time $\times$ group interaction given current sample size was entered in the power analysis and an estimation of power reached with target sample size was performed.

\section{RESULTS}

Participants

For a CONSORT diagram of participant flow, see Fig. 1. Of the 56 included patients, 4 dropped out at screening $(N=2$ assigned to sham, $N=2$ assigned to rTMS) and 7 did not complete treatment $(N=4$ assigned to rTMS, $N=3$ assigned to sham). Baseline characteristics and measures are given in Table 2. There were no significant differences between rTMS and sham groups in measures obtained during the screening, except for MMSE, where a statistically significant, but not clinically meaningful difference was found. Dropouts had significantly higher baseline AUDIT scores (mean $=29, \mathrm{SD}=5.6, P=0.04$ ), but not other significant differences were observed for all baseline measures presented in Table $2(P>0.1)$. Five participants used disulfiram during the last follow-up phase, and two of those during the last two follow-up phases. The average intensity of stimulation in the present study was about $60 \%$ of the total power output of the stimulator (mean $=61 \%$, SEM $=2.3$ ). Facial twitches were common during both rTMS and sham session and 23 of the participants (equally distributed across sham and rTMS sessions) reported feeling moderate to strong headaches after the session. There was no difference between sham and active groups in whether they believed they received active stimulation, assessed using the SETS questionnaire $(x(1)=0.36, P=0.5$; for sham $N=20 / 22$, for rTMS $N=21 / 22$ ).

\section{Alcohol use}

Overall, alcohol use decreased significantly over time, both when estimated by self-report and when analyzing the objective biomarker, but the decrease in use was independent of group (Fig. 2).

Specifically, a significant main effect of time during the course of treatment was observed on both PEth $[N=23$ rTMS, $N=$ 20 sham, $\left.F(1.18,48.6)=10.3, P<0.001, \eta^{2} p=0.2\right]$ and TLFB $[N=$ 22 rTMS, $N=22$ sham, $\left.F(1,42)=50.2, P<0.001, \eta^{2} p=0.54\right]$, 
Table 2. Baseline descriptive statistics

\begin{tabular}{|c|c|c|c|}
\hline & rTMS (mean (SD) & Sham (mean (SD) & $P$ value \\
\hline Age & $50.6(10.4)$ & $53.5(7.5)$ & $U=213, P=0.36$ \\
\hline ADS & $19.3(8.2)$ & $16.7(6.8)$ & $t(43)=1.1, P=0.27$ \\
\hline PEth & $0.9(0.6)$ & $1.1(1.4)$ & $U=238, P=0.93$ \\
\hline MMSE & $28.6(1.1)$ & $29.3(0.8)$ & $U=160, P=0.03$ \\
\hline \multicolumn{4}{|l|}{ ASI } \\
\hline Employment & $0.4(0.3)$ & $0.4(0.3)$ & $U=231, P=0.61$ \\
\hline Medical & $0.3(0.3)$ & $0.2(0.3)$ & $U=199, P=0.21$ \\
\hline Psych & $0.3(0.2)$ & $0.2(0.2)$ & $U=204, P=0.27$ \\
\hline \multicolumn{4}{|l|}{ CPRS-SA } \\
\hline Depression & $8.2(5.3)$ & $7.9(4.8)$ & $U=250, P=0.95$ \\
\hline Anxiety & $7.0(5.2)$ & $6.9(3.9)$ & $U=244, P=0.85$ \\
\hline \multicolumn{4}{|l|}{ NEO-FFI } \\
\hline Neuroticism & $23.7(9.7)$ & $19.4(10.6)$ & $t(43)=1.4, P=0.16$ \\
\hline Extraversion & $22.1(5.9)$ & $25.1(7.3)$ & $t(43)=-1.5, P=0.14$ \\
\hline Openness & $25.3(6.2)$ & $25.4(7.5)$ & $t(43)=-0.05, P=0.96$ \\
\hline Agreeableness & $33.5(5.9)$ & $31.9(6.7)$ & $t(43)=0.8, P=0.41$ \\
\hline Conscientiousness & $26.8(7.5)$ & $30.2(7.7)$ & $t(43)=-1.5, P=0.15$ \\
\hline
\end{tabular}

indicating decreased drinking during treatment, irrespective of whether participants received rTMS of sham stimulation. For TLFB there was an effect of time also during follow-up period $[N=21$ rTMS, $N=18$ sham, $\left.F(4.1,150.2)=6.3, \quad P<.001, \eta^{2} p=0.15\right]$, indicating that compared to screening, self-reports of alcohol consumption were lower independently of whether participants received rTMS or sham.

No main effect of group was observed on consumption as assessed by PEth during treatment and follow-up (all $P \mathrm{~s}>0.3$ ), nor was there a time $\times$ group interaction during treatment $(P=0.6)$ and follow-up ( $N=14$ rTMS, $N=14$ sham, $P=0.8$, Fig. 2). In agreement with the biomarker (PEth) data, there was also no main effect of group on self-reported use assessed with TLFB during treatment or follow-up $\left(P_{S}>0.3\right)$, nor was there a significant time $\times$ group interaction during treatment and followup (Ps > 0.4); Fig. 2).

PETth and TLFB results in the rTMS treatment group were not affected by stimulation strength (Ps $>0.7)$ nor by insular depth (Ps > 0.6).

An interim analysis was carried out on PEth results. The time $x$ treatment interaction on PEth scores during treatment phase, achieved an observed power of 0.098 , given current sample size of 43 participants. Achieved power was estimated given target sample size $(N=82)$, empirically assessed correlation among measures $(r=0.5)$, and current effect size of $f=0.047$. The analysis estimated a power of 0.14 , indicating that even with double sample size, the power of our statistics would have remained largely below our target. In order to achieve a power of 0.8 given the current effect size, we would have needed to collect a total of 620 participants. This evidence led to the decision to terminate the live phase of the study, and initiate final data analysis.

Alcohol craving

Acute and retrospective self-reports of alcohol craving revealed a general decrease during treatment, which was independent of group (Fig. 2).

There was a significant main effect of time during treatment, for both AUQ $[N=20 \mathrm{rTMS}, N=22$ sham, $F(4.35,174.1)=17.27, P<$ $\left.0.001, \eta^{2} p=0.3\right]$ and PACS $[N=22$ rTMS, $N=22$ sham, $F(1.4,59.2)$ $=5.3, P=0.01, \eta^{2} p=0.12$ ]. However, no between group effect was found in all measures (all $P S>0.1$ ), and no significant time* ${ }^{*}$ roup could be found in neither AUQ during treatment $(P=0.4)$ nor for PACS during treatment and follow-up sessions $(P=0.6, P=0.4)$.

Psychiatric ratings, inflammation biomarkers and nicotine consumption

Psychiatric symptoms also showed a significant decrease over time. CPRS-SA results, revealed a significant long-term decrease in self-report measures of depression and anxiety [depression $N=15$ rTMS, $N=18$ sham, $F(4.13,127.9)=6.14, P<0.001, \eta^{2} p=0.2$; anxiety $N=12$ rTMS, $N=17$ sham, $F(3.47,93.8)=10.16, P<0.001$, $\eta^{2} p=0.3$ ] (Fig. S1). Similarly to other measures, no group effects were observed (all $P s>0.6$ ) together with no time $\times$ group interaction (all $P \mathrm{~s}>0.4$ ). CGI scores decreased during treatment $\left[N=22\right.$ rTMS, $N=22$ sham, $F(1.89,79.5)=7.64, P=0.001, \eta^{2}$ 

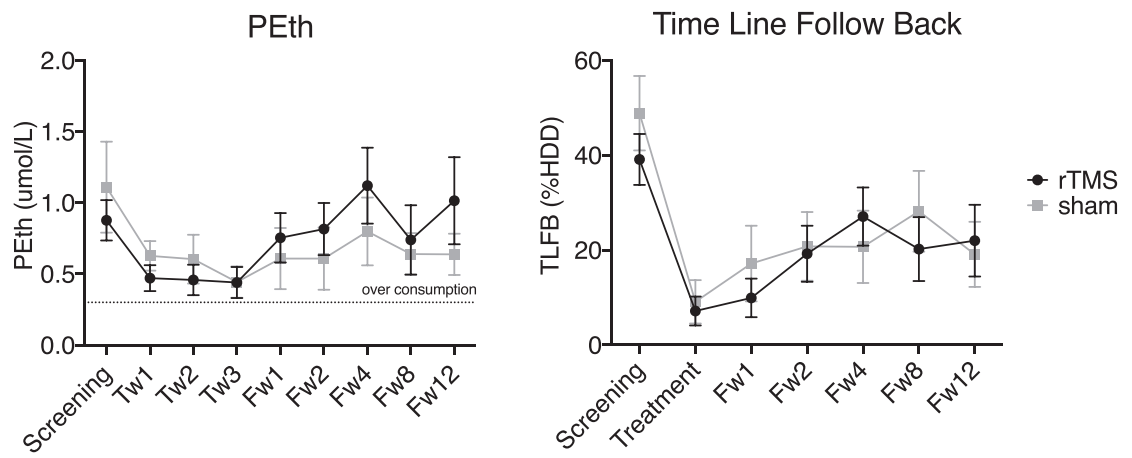

AUQ alcohol

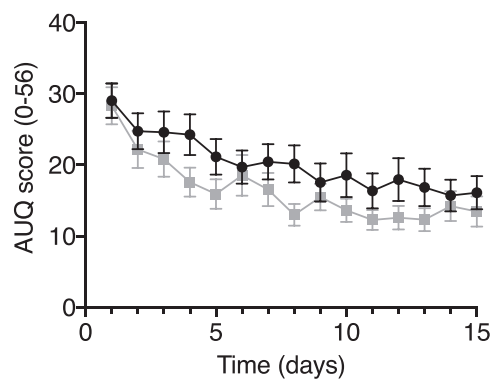

PACS Score

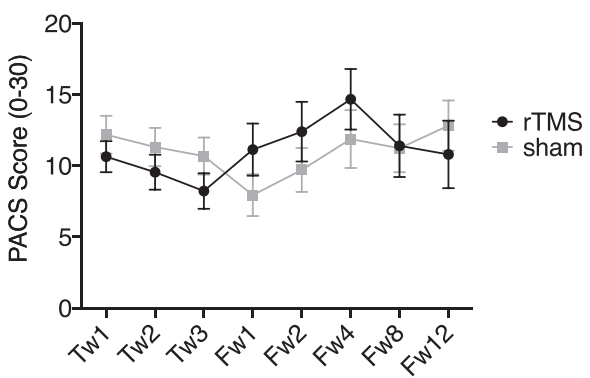

Fig. 2 Average consumption and craving scores measured in rTMS and sham groups at screening, treatment and follow-up visits. Upper level: Consumption as measured by PEth and TLFB. For PEth, two separate ANOVAs were performed. In the first $2 \times 4$ ANOVA, the "time" factor included four levels, corresponding to screening and treatment weeks 1,2 , and 3 . In the second ANOVA, the "time" factor included six levels (screening and all follow-up visits). For TLFB a $2 \times 2$ ANOVA was performed using scores collected at screening and end of treatment. In addition, a $2 \times 6$ ANOVA was performed on screening and all follow-up visits. A significant effect of time during treatment period was observed in both measures, independently of group. Lower level: craving scores by AUQ and PACS. For AUQ scores, a $2 \times 15$ ANOVA was performed, the factor "time" included each day when AUQ was collected. PACS scores were entered in a $2 \times 3$ ANOVA, where the withinsubject "time" factor included assessments at treatment weeks 1, 2, and 3. Error bars show standard error of the mean. There was a significant decrease in craving and drinking during treatment in all measures, independently of group. Tw $[n]=$ week $n$ of treatment; Fw $[n]=$ follow-up week $\mathrm{n}$. Upper right: Treatment = the part of TLFB from the first follow-up visit covering the weeks of rTMS treatment. \%HDD, percent heavy drinking days

$p=0.1]$, but not at follow-up $(P=0.5)$. No significant differences were found between treatment groups in inflammation and neurotrophic factor biomarkers (all Ps $>0.1$, see Supplemental Material, Table S1). The proportion of subject who consumed nicotine did not differ between groups neither at baseline or at any follow-up timepoints (all $X \mathrm{~s}<1.38$, all $P \mathrm{~s}>0.3$; baseline: sham $N=22 / 26$, rTMS $N=22 / 25$; Fw1: $\operatorname{sham} N=14 / 18$, rTMS $N=12 / 20$; Fw2: $\operatorname{sham} N=14 / 21$ rTMS $N=15 / 20 ;$ Fw4: sham $N=13 / 20$, rTMS $N=13 / 18$; Fw8: sham $N=12 / 21$, rTMS $N=16 / 22$; Fw12: sham $N=17 / 24$, rTMS 12/19).

\section{MRI findings}

Resting state. The resting-state seed-based correlation analysis revealed a significant group effect in connectivity at follow-up for bilateral posterior insula (PI) seeds (Fig. 3, $N=18$ rTMS, $N=$ 21 sham, per-voxel $P<0.002$, cluster corrected at $a=0.05$ ). Both groups revealed a positive correlation between right PI and left precuneus; however, the correlation was significantly lower in the rTMS group [Fig. 3b, rTMS < sham, $\mathrm{MNI}=1,-23,31$, cluster size $=$ $\left.8, F(1,36)=22.88, P<0.001 \eta^{2} p=0.4\right]$. Correlation between left PI and right cingulate was significantly different between groups with positive correlations scores in the rTMS group and negative for the sham group $[N=18 \mathrm{rTMS}>N=20$ sham, $\mathrm{MNI}-11,-71$, 28 , cluster size $8, F(1,36)=15.88, P<0.001, \eta^{2} p=0.3$ ] (Fig. 3b). $\beta$ Correlation coefficients scores in the rTMS treatment group were not affected by insular depth $(P S>0.1)$.

Monetary incentive delay task. A main effect of anticipation for all four of the factorial analyses replicated previous findings from the literature and revealed significant clusters in regions including striatum, insula, thalamus, and medial wall premotor regions (Fig. S2, $N=18 \mathrm{rTMS}, N=21$ sham, per-voxel $P=0.002$, cluster size threshold $=11$ ). However, no significant group nor group $\times$ stimuli interaction effects were observed. Activation peaks for each factorial analysis are presented in Table S3.

Negative-affect picture processing. A main effect of stimulus across groups revealed significantly increased activity in several regions including the insular cortex bilaterally to face pictures, and increased activity in the lingual gyrus to pictures from The International Affective Picture System (IAPS) (Fig. S3 and Table S4, $N=17$ rTMS, $N=21$ sham, per-voxel $P=0.002$, cluster size threshold $=11$ ). No significant group nor stimuli $\times$ group interactions were identified. A $2 \times 2$ ANOVA on the extracted $\beta$ values in the insula confirmed a significant main effect of stimulus [left anterior insula: $F(1,36)=31.90, P<0.001, \eta^{2} p=0.47$; right anterior insula: $F(1,36)=32.23, P<0.001, \eta^{2} p=0.47$, driven by face pictures (left anterior insula: $t=-5.57, P<0.001$; right anterior insula: $t=-5.58, P<0.001)$, and a non-significant stimuli $\times$ group interaction $(P S>0.2)$.

Alcohol beverage picture processing. Alcohol pictures compared to non-alcohol pictures activated significantly more the visual cortex (MNI 4, -83, -5) and left precuneus (MNI -14, -89, 4) (pervoxel $P=0.002$, cluster size threshold $=12$ ). However, the $2 \times 2$ ANOVA showed no significant group and stimuli $\times$ group interaction $(N=18$ rTMS, $N=20$ sham). 


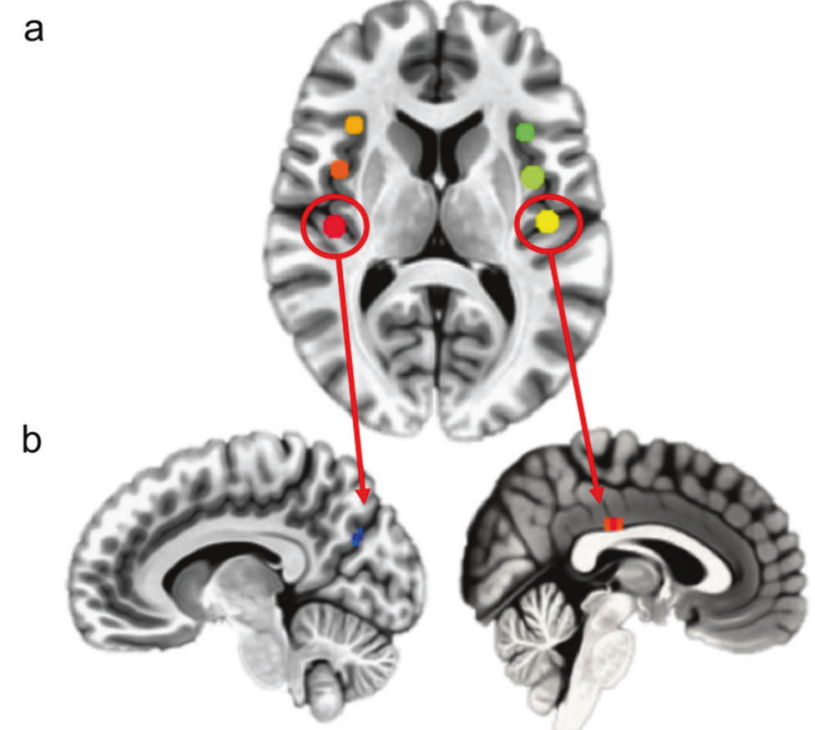

Left Precuneus
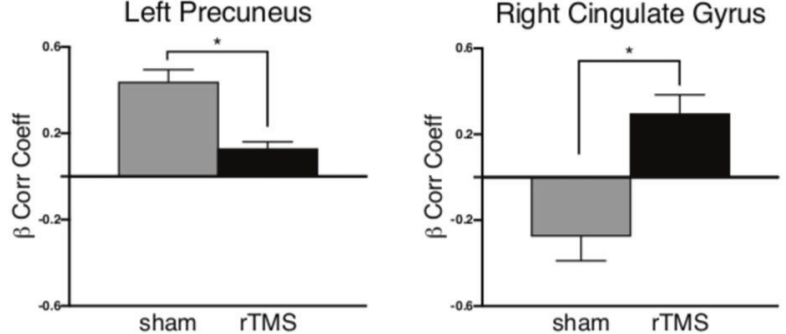

Fig. 3 Resting-state connectivity analysis at follow-up. a Insula seed locations identified by maximizing the bilateral connectivity of each seed location. b Brain regions showing significantly different correlation scores in rTMS and sham groups between right PI seed regions and left precuneus and left $\mathrm{PI}$ seed and right posterior cingulate (per-voxel $P<0.002$, cluster corrected at $a=0.05$ ). The rTMS group presented a significantly lower connectivity between left $\mathrm{PI}$ and left precuneus [rTMS $<$ sham, $\mathrm{MNI}=1,-23,31$, cluster size $\left.=8, F(1,36)=22.88, P<0.001 \eta^{2} p=0.384\right]$ and a significantly greater connectivity between left $\mathrm{PI}$ and right cingulate $[N=18$ rTMS $>N=20$ sham, MNI $-11,-71,28$, cluster size $8, F(1,36)=$ 15.88, $\left.P<0.001 \eta^{2} p=0.3\right]$

\section{DISCUSSION}

rTMS is currently receiving considerable interest as an addiction treatment [9], but evidence available to date does not provide sufficient support for the efficiency of rTMS in treatment of alcoholism [13]. A small number of studies have relied on randomized controlled methodology considered standard when evaluating pharmacological interventions. Here, we carried out a double-blind, sham-controlled randomized trial to investigate whether rTMS targeting the insula can produce persistent neuromodulatory effects with a clinically meaningful impact on alcohol craving and use. Although rsMRI analysis provided some support for the ability of rTMS to modulate insula function in a measurable manner, a broad range of objective, and self-reported outcome measures did not support that this effect translated into decreased alcohol craving or use.

An established role of the insula in craving across drug categories [21] provided a strong mechanistic foundation for our study. With its heterogeneous architecture and the wide degree of connections organized according to a posterior-anterior gradient $[51,52]$, the insula is a highly integrative yet functionally specialized region [53]. It has been theorized that the anterior portion of the insula represents a final stage of integration, in which interoceptive information is progressively gathered "into" awareness [54]. This model is consistent with the identification of the anterior insula as a major hub of the salience network, a network of critical importance in attention and awareness [55]. The connection between anterior insula with premotor regions in the mid-cingulate cortex (another key node of the salience network) highlight its influence on behavior, with important implication in craving and addiction [56].

Our stimulation protocol paralleled that used in a prior positive trial of rTMS in nicotine addiction [12], but used an $\mathrm{H} 8$ coil that more selectively targeted the insula (and by necessity overlaying superficial regions), while excluding rostral prefrontal regions. Primary outcome measures included heavy drinking, as determined by structured and validated self-reports of drinking assessed with TLFB methodology that is standard in Food and Drug Administrationregulated trials of alcoholism treatments, but also PEth, an objective, highly selective and quantitative biomarker of alcohol use. Uniquely among rTMS studies in addiction to date, we combined assessment of these clinical outcomes with functional resting-state and taskbased brain measures to establish whether our intervention did in fact modulate function of the targeted brain structure in a measurable way.

Using this stringent design, rTMS did not affect how strongly the subjects craved to drink, nor did it influence how much alcohol they actually consumed. Nor it affected nicotine consumption. The significant effect of rTMS stimulation in restingstate connectivity between bilateral $\mathrm{PI}$ and precuneus/posterior cingulate cortex suggests that rTMS might have introduced changes in cortical connectivity, but that this occurred without affecting addiction-related behavior. The lack of stimulationdriven behavioral effect could be due to the fact that rTMS stimulation might have reached the insula only partially, or at insufficient intensity. However, several patients presented facial muscle twitches induced by the TMS stimulation at surface $(\sim 138$ $\mathrm{V} / \mathrm{m}$ ). This highlights the challenges that deep TMS studies suffer in balancing the need to reach a sufficient electric field intensity in deeper structures while containing patient discomfort. Alternatively, it is possible that concomitant stimulation of the insula and the PFC, as was done in Dinur-Klein et al. [12], is required in order to induce effects on addictive behavior, while in this study the PFC was not stimulated effectively. Cue exposure of the kind used during our TMS treatment has been suggested as a therapeutic intervention to decrease alcohol use. If effective in that regard, it could potentially decrease the sensitivity to detect a TMS effect through a floor effect. However, a meta-analysis of cue exposure therapy does not support its efficacy in alcohol addiction [57], while the nicotine addiction study by Dinur-Klein et al. [12] on which we based our design in fact indicated that cue exposure increased, rather than decreased the TMS effect.

Together with alcohol craving and consumption measures, we examined blood oxygen level-dependent (BOLD) brain responses during tasks that typically involve insula activity. First, we addressed whether rTMS influenced reward processing using the classic MID task [27]. There were no significant brain differences between the rTMS and sham groups during anticipation of reward or punishment. In both groups, we identified regions typically associated with processing the anticipation of reward and punishment, such as insula, caudate nucleus, putamen, and thalamus.

Our findings of activation during reward anticipation parallel those confirmed for healthy volunteers by a recent activationlikelihood estimate (ALE) meta-analysis of some 50 studies in healthy volunteers [58]. When compared to controls, it has been reported that alcohol-dependent patients show altered activity in ventral striatum on this task, although the directionality of the altered response is a matter of debate [59]. In the present study, we did not compare alcoholics with healthy participants, and our data do not, therefore, address this issue. Our ability to replicate these responses while complying with current stringent statistical 
standards [49] suggests that the negative findings of a treatment effect do not reflect a methodological artifact.

We found the same pattern for the other tasks, which included negative-affect picture and alcohol-related picture processing. For negative-affect picture processing, the insular cortex was significantly more activated to faces depicting negative facial expressions of fear and anger compared to negative-affect pictures from the IAPS catalog, reflecting increased salience processing towards faces [60]. The visual cortex and lingual gyrus were activated for the opposite contrast, probably due to greater detail heterogeneity for IAPS compared to faces pictures. Alcohol-related pictures compared to non-alcohol-related pictures also triggered activity in the visual cortex, indicating increased attention towards alcohol pictures [61].

Similar to the vast majority of RCTs aimed at evaluating therapeutic interventions, we did not include a healthy control group. Nevertheless, the baseline imaging measures obtained in our patient sample would be of interest to compare against a group of healthy controls. This comparison is in progress through a meta-analysis within the EU-funded SyBil-AA consortium, of which the present study is a part.

In summary, although the rsMRI analysis provides some support for the ability of our rTMS intervention to exert measurable neuromodulatory effects on insula function, this did not affect alcohol craving or drinking, nor did it affect task-based brain responses. Strategies for the future may include attempts to increase stimulation intensity and extend the duration of treatment, as well as explore other targets, such as the anterior cingulate cortex. Similar to what is typically found in medication trials of alcohol addiction, we found a large, statistically significant decline in alcohol consumption and craving during treatment irrespective of group allocation. Two factors may have contributed to the large size of this effect. First, treatment delivery involved interactions with qualified nursing staff daily for 3 weeks, a frequency higher than that in medication trials. Second, the daily induction of alcohol cravings through the use of alcohol cues amounts to provocation with response inhibition, an established cognitive behavioral therapy method to extinguish undesirable responses that under the name Cue Exposure Therapy has support for some efficacy of its own [57]. The overall reduction in drinking was accompanied by improvements in depression and anxiety (CPRS) that lasted up to 3 months follow-up, illustrating the beneficial effects of reductions in drinking for a broad range of psychiatric outcomes.

\section{FUNDING AND DISCLOSURE}

This research was supported by grant 2013-07434 from The Swedish Research Council and by grant 668863 -SyBil-AA from the European Union's Horizon 2020 research and innovation programme. $\mathrm{MH}$ has received research funding from, and is on a scientific advisory board for BrainsWay Inc. AZ is an inventor of deep TMS coils and has financial interest in BrainsWay which produces and markets these coils. All the other authors declare no competing interests.

\section{ACKNOWLEDGEMENTS}

We thank Åsa Axén for outstanding management of research participants and Andrew Wold for aid in TMS stimulation. The authors gratefully acknowledge staff at the Center for Medical Imaging and Visualization (CMIV), Linköping University Hospital, Sweden.

\section{ADDITIONAL INFORMATION}

Supplementary Information accompanies this paper at (https://doi.org/10.1038/ s41386-019-0565-7).

Publisher's note Springer Nature remains neutral with regard to jurisdictional claims in published maps and institutional affiliations.

\section{REFERENCES}

1. Compton WM, Dawson DA, Goldstein RB, Grant BF. Crosswalk between DSM-IV dependence and DSM-5 substance use disorders for opioids, cannabis, cocaine and alcohol. Drug Alcohol Depend. 2013;132:387-90.

2. Berglund $M$, Thelander S, Salaspuro M, Franck J, Andreasson S, Ojehagen A. Treatment of alcohol abuse: an evidence-based review. Alcohol Clin Exp Res. 2003;27:1645-56.

3. Jonas DE, Amick HR, Feltner C, Bobashev G, Thomas K, Wines R. et al. Pharmacotherapy for adults with alcohol use disorders in outpatient settings: a systematic review and meta-analysis. JAMA. 2014;311:1889-900.

4. Sinha R. New findings on biological factors predicting addiction relapse vulnerability. Curr Psychiatry Rep. 2011;13:398-405.

5. Mark TL, Kassed CA, Vandivort-Warren R, Levit KR, Kranzler HR. Alcohol and opioid dependence medications: prescription trends, overall and by physician specialty. Drug Alcohol Depend. 2009;99:345-9.

6. Brunoni AR, Chaimani A, Moffa AH, Razza LB, Gattaz WF, Daskalakis ZJ, et al. Repetitive transcranial magnetic stimulation for the acute treatment of major depressive episodes: a systematic review with network meta-analysis. JAMA Psychiatry. 2017;74:143-52.

7. Rehn S, Eslick GD, Brakoulias V. A meta-analysis of the effectiveness of different cortical targets used in repetitive transcranial magnetic stimulation (rTMS) for the treatment of obsessive-compulsive disorder (OCD). Psychiatr Q. 2018;89:645-65.

8. Yan T, Xie Q, Zheng Z, Zou K, Wang L. Different frequency repetitive transcranial magnetic stimulation (rTMS) for posttraumatic stress disorder (PTSD): a systematic review and meta-analysis. J Psychiatr Res. 2017;89:125-35.

9. Diana M, Raij T, Melis M, Nummenmaa A, Leggio L, Bonci A. Rehabilitating the addicted brain with transcranial magnetic stimulation. Nat Rev Neurosci. 2017; 18:685-93.

10. Feil J, Zangen A. Brain stimulation in the study and treatment of addiction. Neurosci Biobehav Rev. 2010;34:559-74.

11. Amiaz R, Levy D, Vainiger D, Grunhaus L, Zangen A. Repeated high-frequency transcranial magnetic stimulation over the dorsolateral prefrontal cortex reduces cigarette craving and consumption. Addiction (Abingdon, Engl). 2009;104:653-60.

12. Dinur-Klein L, Dannon $P$, Hadar A, Rosenberg $O$, Roth $Y$, Kotler $M$, et al. Smoking cessation induced by deep repetitive transcranial magnetic stimulation of the prefrontal and insular cortices: a prospective, randomized controlled trial. Biol Psychiatry. 2014;76:742-9.

13. Maiti R, Mishra BR, Hota D. Effect of high-frequency transcranial magnetic stimulation on craving in substance use disorder: a meta-analysis. J Neuropsychiatry Clin Neurosci. 2017;29:160-71.

14. Li X, Hartwell KF, Owens M, Lematty T, Borckardt JJ, Hanlon CA, et al. Repetitive transcranial magnetic stimulation of the dorsolateral prefrontal cortex reduces nicotine cue craving. Biol Psychiatry. 2013;73:714-20.

15. Mishra BR, Nizamie SH, Das B, Praharaj SK. Efficacy of repetitive transcranial magnetic stimulation in alcohol dependence: a sham-controlled study. Addiction (Abingdon, Engl). 2010;105:49-55.

16. Höppner J, Broese T, Wendler L, Berger C, Thome J. Repetitive transcranial magnetic stimulation (rTMS) for treatment of alcohol dependence. World J Biol Psychiatry. 2011;12:57-62.

17. Addolorato G, Antonelli M, Cocciolillo F, Vassallo GA, Tarli C, Sestito L, et al. Deep transcranial magnetic stimulation of the dorsolateral prefrontal cortex in alcohol use disorder patients: effects on dopamine transporter availability and alcohol intake. Eur Neuropsychopharmacol. 2017;27:450-61.

18. Ceccanti $M$, Inghilleri $M$, Attilia ML, Raccah R, Fiore $M$, Zangen $A$, et al. Deep TMS on alcoholics: effects on cortisolemia and dopamine pathway modulation. A pilot study. Can J Physiol Pharmacol. 2015;93:283-90.

19. Kedzior KK, Gerkensmeier I, Schuchinsky M. Can deep transcranial magnetic stimulation (DTMS) be used to treat substance use disorders (SUD)? A systematic review. BMC Psychiatry. 2018;18:137.

20. Bellamoli E, Manganotti P, Schwartz RP, Rimondo C, Gomma M, Serpelloni G. rTMS in the treatment of drug addiction: an update about human studies. Behav Neurol. 2014;2014:815215.

21. Garavan H. Insula and drug cravings. Brain Struct Funct. 2010;214:593-601.

22. Bonson KR, Grant SJ, Contoreggi CS, Links JM, Metcalfe J, Weyl HL, et al. Neural systems and cue-induced cocaine craving. Neuropsychopharmacology. 2002;26: 376-86.

23. Pelchat $M L$, Johnson $A$, Chan R, Valdez J, Ragland JD. Images of desire: foodcraving activation during fMRI. Neuroimage. 2004;23:1486-93.

24. Wang Z, Faith M, Patterson F, Tang K, Kerrin K, Wileyto EP, et al. Neural substrates of abstinence-induced cigarette cravings in chronic smokers. J Neurosci. 2007;27:14035-40.

25. Naqvi $\mathrm{NH}$, Bechara A. The insula and drug addiction: an interoceptive view of pleasure, urges, and decision-making. Brain Struct Funct. 2010;214:435-50.

26. Naqvi $\mathrm{NH}$, Rudrauf $\mathrm{D}$, Damasio $\mathrm{H}$, Bechara $\mathrm{A}$. Damage to the insula disrupts addiction to cigarette smoking. Science. 2007;315:531-34. 
27. Knutson B, Westdorp A, Kaiser E, Hommer D. FMRI visualization of brain activity during a monetary incentive delay task. Neuroimage. 2000;12:20-7.

28. Hariri AR, Tessitore A, Mattay VS, Fera F, Weinberger DR. The amygdala response to emotional stimuli: a comparison of faces and scenes. Neuroimage. 2002;17:317-23.

29. APA. Diagnostic and Statistical Manual of Mental Disorders. 4th ed. Text Revision. Washington, DC: APA; 2000.

30. Tombaugh TN, Mclntyre NJ. The mini-mental state examination: a comprehensive review. J Am Geriatr Soc. 1992;40:922-35.

31. Rossi S, Hallett M, Rossini PM, Pascual-Leone A. Safety, ethical considerations, and application guidelines for the use of transcranial magnetic stimulation in clinical practice and research. Clin Neurophysiol. 2009;120:2008-39.

32. Saunders JB, Aasland OG, Babor TF, de la Fuente JR, Grant M. Development of the Alcohol Use Disorders Identification Test (AUDIT): WHO Collaborative Project on early detection of persons with harmful alcohol consumption-Il. Addiction (Abingdon, Engl). 1993;88:791-804.

33. Skinner HA, Allen BA. Alcohol dependence syndrome: measurement and validation. J Abnorm Psychol. 1982;91:199-209.

34. McLellan AT, Cacciola JC, Alterman Al, Rikoon SH, Carise D. The Addiction Severity Index at 25: origins, contributions and transitions. Am J Addict. 2006;15:113-24.

35. Rosen CS, Henson BR, Finney JW, Moos RH. Consistency of self-administered and interview-based Addiction Severity Index composite scores. Addiction (Abingdon, Engl). 2000;95:419-25

36. First MB, Gibbon M, Spitzer RL, Williams JBW. Structured clinical interview for DSM-IV axis I disorders, clinician version (SCID-CV). Washington, DC: American Psychiatric Press; 1996.

37. Svanborg P, Asberg M. A new self-rating scale for depression and anxiety states based on the Comprehensive Psychopathological Rating Scale. Acta Psychiatr Scand. 1994;89:21-8.

38. Guy W. ECDEU assessment manual for psychopharmacology. Rockville, MD: US Dept. of Health, Education, and Welfare, Public Health Service, Alcohol, Drug Abuse, and Mental Health Administration, National Institute of Mental Health, Psychopharmacology Research Branch, Division of Extramural Research Programs; 1976.

39. Costa PT, McCrae RR. Revised NEO Personality Inventory (NEO PI-R) and NEO Fivefactor Inventory (NEO-FFI): professional manual. Odessa, FL: Psychological Assessment Resources; 1992.

40. Zangen $A$, Roth $Y$, Voller $B$, Hallett $M$. Transcranial magnetic stimulation of deep brain regions: evidence for efficacy of the H-coil. Clin Neurophysiol. 2005;116:775-79.

41. Zibman S, Pell GS, Barnea-Ygael N, Roth Y, Zangen A. Application of transcranial magnetic stimulation for major depression: coil design and neuroanatomical variability considerations. Eur Neuropsychopharmacol. 2019; https://doi.org/ 10.1016/j.euroneuro.2019.06.009 (1873-7862 (Electronic)).

42. Wurst FM, Thon N, Yegles M, Schruck A, Preuss UW, Weinmann W. Ethanol metabolites: their role in the assessment of alcohol intake. Alcohol Clin Exp Res. 2015;39:2060-72.
43. Sobell LC, Sobell MB. Timeline follow-back. In: Allen RZLJP, editor. Measuring alcohol consumption: psychosocial and biochemical methods. Totowa, NJ: Humana Press; 1992. p. 41-72.

44. Bohn MJ, Krahn DD, Staehler BA. Development and initial validation of a measure of drinking urges in abstinent alcoholics. Alcohol Clin Exp Res. 1995;19:600-6.

45. Younger J, Gandhi V, Hubbard E, Mackey S. Development of the Stanford Expectations of Treatment Scale (SETS): a tool for measuring patient outcome expectancy in clinical trials. Clin Trials. 2012;9:767-76.

46. Flannery BA, Volpicelli JR, Pettinati HM. Psychometric properties of the Penn Alcohol Craving Scale. Alcohol Clin Exp Res. 1999;23:1289-95.

47. Cox RW. AFNI: software for analysis and visualization of functional magnetic resonance neuroimages. Comput Biomed Res Int J. 1996;29:162-73.

48. Eklund A, Nichols TE, Knutsson $\mathrm{H}$. Cluster failure: Why fMRI inferences for spatial extent have inflated false-positive rates. Proc Natl Acad Sci USA. 2016;113:7900-5.

49. Cox RW, Chen G, Glen DR, Reynolds RC, Taylor PA. fMRI clustering and falsepositive rates. Proc Natl Acad Sci USA. 2017;114:E3370-71.

50. Rasband WS. ImageJ: image processing and analysis in Java. Astrophysics Source Code Library; 2012.

51. Augustine JR. Circuitry and functional aspects of the insular lobe in primates including humans. Brain Res Brain Res Rev. 1996;22:229-44.

52. Cerliani L, Thomas RM, Jbabdi S, Siero JC, Nanetti L, Crippa A, et al. Probabilistic tractography recovers a rostrocaudal trajectory of connectivity variability in the human insular cortex. Hum Brain Mapp. 2012;33:2005-34.

53. Kurth $F$, Zilles $K$, Fox PT, Laird AR, Eickhoff SB. A link between the systems: functional differentiation and integration within the human insula revealed by meta-analysis. Brain Struct Funct. 2010;214:519-34.

54. Craig AD. How do you feel-now? The anterior insula and human awareness. Nat Rev Neurosci. 2009;10:59-70.

55. Menon V, Uddin LQ. Saliency, switching, attention and control: a network model of insula function. Brain Struct Funct. 2010;214:655-67.

56. Paulus MP. Neural basis of reward and craving - a homeostatic point of view. Dialog Clin Neurosci. 2007;9:379-87.

57. Mellentin Al, Skot L, Nielsen B, Schippers GM, Nielsen AS, Stenager E, et al. Cue exposure therapy for the treatment of alcohol use disorders: a meta-analytic review. Clin Psychol Rev. 2017;57:195-207.

58. Oldham S, Murawski C, Fornito A, Youssef G, Yucel M, Lorenzetti V. The anticipation and outcome phases of reward and loss processing: a neuroimaging meta-analysis of the monetary incentive delay task. Hum Brain Mapp. 2018;39:3398-418.

59. Balodis IM, Potenza MN. Anticipatory reward processing in addicted populations: a focus on the monetary incentive delay task. Biol Psychiatry. 2015;77:434-44.

60. Fan J, Gu X, Liu X, Guise KG, Park Y, Martin L. et al. Involvement of the anterior cingulate and frontoinsular cortices in rapid processing of salient facial emotional information. Neuroimage. 2011;54:2539-46.

61. Schupp HT, Junghofer M, Weike Al, Hamm AO. The selective processing of briefly presented affective pictures: an ERP analysis. Psychophysiology. 2004;41:441-9. 